\title{
Magnetic field resilient superconducting fractal resonators for coupling to free spins
}

\author{
S. E. de Graaf, ${ }^{\text {a) }}$ A. V. Danilov, A. Adamyan, T. Bauch, and S. E. Kubatkin \\ Department of Microtechnology and Nanoscience, MC2, Chalmers University of Technology, \\ SE-41296 Goteborg, Sweden
}

(Received 12 October 2012; accepted 10 November 2012; published online 18 December 2012)

\begin{abstract}
We demonstrate a planar superconducting microwave resonator intended for use in applications requiring strong magnetic fields and high quality factors. In perpendicular magnetic fields of $20 \mathrm{mT}$, the niobium resonators maintain a quality factor above 25000 over a wide range of applied powers, down to single photon population. In parallel field, the same quality factor is observed above $160 \mathrm{mT}$, the field required for coupling to free spins at a typical operating frequency of $5 \mathrm{GHz}$. We attribute the increased performance to the current branching in the fractal design. We demonstrate that our device can be used for spectroscopy by measuring the dissipation from a pico-mole of molecular spins. (C) 2012 American Institute of Physics. [http://dx.doi.org/10.1063/1.4769208]
\end{abstract}

\section{INTRODUCTION}

High quality $(\mathrm{Q})$ factor superconducting resonators have become instrumental in the interrogation of solid state qubits, thanks to their ability to concentrate fields, reaching a good coupling between a quantum system under study and the resonator. ${ }^{1-3}$ Recently, an ensemble of spins embedded in a crystal has become a subject of quantum manipulation by interaction with a superconducting resonator. ${ }^{4,5}$ Spin systems are very promising quantum objects since their coherence times can be very long. However, the magnetic field required to bring free-electron spins into resonance with microwave field exceeds many times the field a standard coplanar waveguide (CPW) resonator can withstand. That is why the first experiments with spins were done on systems with large zero-field splitting from a crystalline field, making them compatible with the readout using superconducting resonators.

Obviously, there is a need for high quality resonators capable of operation in high magnetic fields to widen the choice of spin systems available for quantum measurements. Much attention has been given to studies of coplanar resonators at milliKelvin temperatures in order to pinpoint and eliminate different types of dissipative processes limiting the quality factor. ${ }^{6-8}$ Several methods have been developed to improve the quality factors in magnetic fields, such as antidots in ground planes and the central conductor of the resonator $^{9-13}$ as well as narrow slots in the center conduc$\operatorname{tor}^{14}$ to reduce the degrees of freedom for vortices in the superconductor.

Generally speaking, strong enough magnetic field introduces vortices in the superconductor and can cause depairing of Cooper pairs. Both effects lead to additional dissipation in the AC field of a superconducting resonator and can be described as an extra effective resistance that the superconductor acquires when placed in the magnetic field. ${ }^{15}$ By

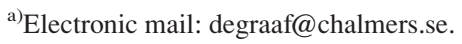

reducing the current in the resonator in regions where this effective resistance is large, it should be possible to reduce the losses caused by magnetic fields.

In this paper, we report design, fabrication, and measurements on niobium fractal superconducting resonators operating at a typical resonance frequency of $f_{0}=5 \mathrm{GHz}$, preserving high internal quality factors of more than $2.5 \times 10^{4}$ in magnetic fields corresponding to the Zeeman splitting for free spins $h f_{0} / 2 \mu_{B}$. We show experimentally and demonstrate theoretically that our fractal design, thanks to its specific current branching and current distribution, works well by reducing losses caused by magnetic fields.

The outline of this paper is the following. First, we describe the design and general characteristics of the fractal resonator. The experimental details are described in Sec. III. In Sec. IV A, we describe the behavior of the resonators in magnetic field and in Sec. IV B we give a theoretical description to why they show significantly reduced loss rates in this regime. Next, in Sec. IVC, we investigate how the ground planes influence the overall behavior in magnetic fields. We find that also here a fractal-like topology is advantageous. We then look into the power dependence (Sec. IV D) of the resonators and find that they suffer more from zero-field loss than conventional coplanar resonators, albeit in magnetic fields this loss is not dominating. Finally, in Sec. IVE, we use the fractal resonator for electron spin resonance (ESR) spectrometry and we measure the dissipation from a microscopic volume of organic molecules containing free radicals, showing that these resonators indeed are useful for free spin interaction.

\section{RESONATOR DESIGN}

In Fig. 1, we show a superconducting resonator made out of a fractal network. At a first glance, it is difficult to get an overview of its structure, but the idea behind it is clarified in Fig. 2. Starting from a half-wavelength coplanar resonator, we turn it into a U-shape and remove the ground plane between the "prongs," so that the mutual capacitance between 


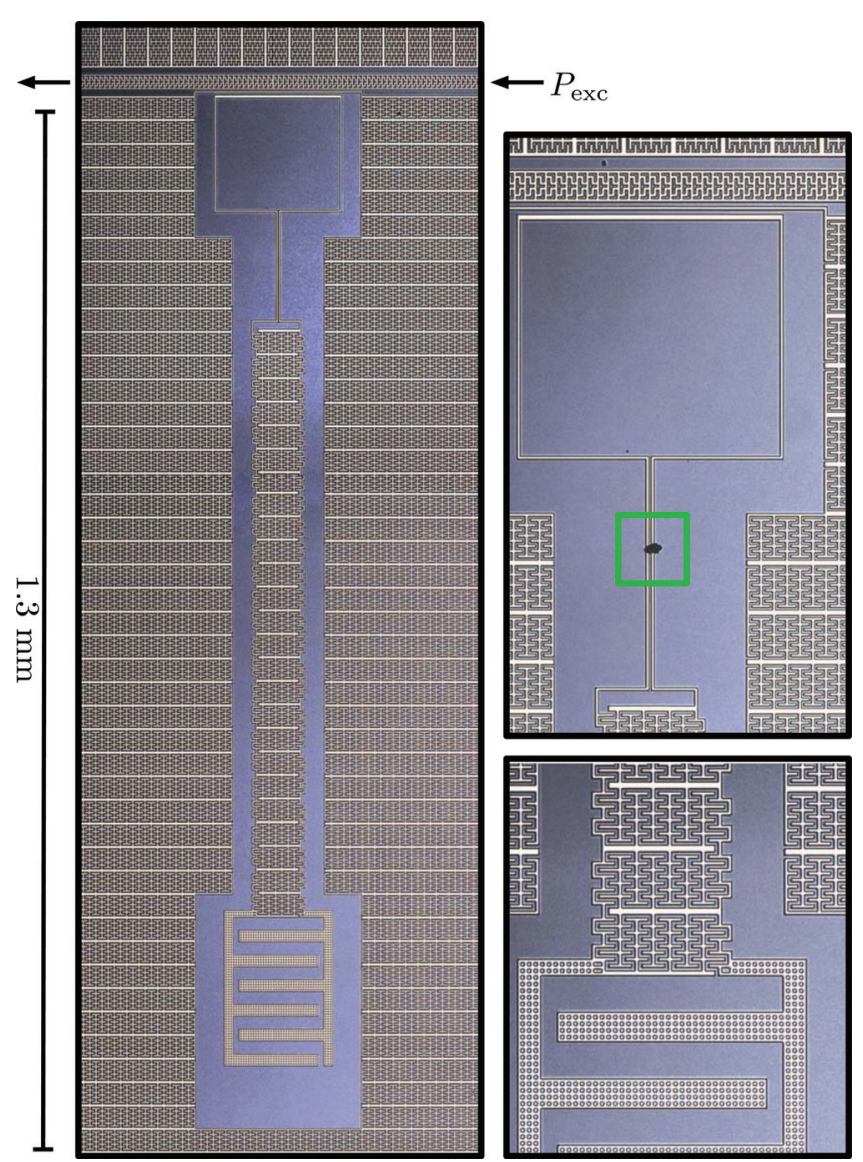

FIG. 1. Optical image of one of the resonators used in this study. Both metalized areas (light grey) and gaps have a typical dimension of $2 \mu \mathrm{m}$. The ground plane has the same structure as the resonator itself. The prongs are terminated with a wide gap interdigital capacitor so that the resonance frequency can be tuned by covering the terminal area with a dielectric plate. Top right and bottom right panels are close ups on the two ends of the resonator. Green square indicates the location of the inset in Fig. 9.

the prongs becomes important (see Fig. 2(b)). Such a structure supports a resonance with a current distribution sketched in Fig. 2(a), very similar to the traditional $\lambda / 2$ resonance. We can increase the mutual capacitance per unit length between the prongs by creating an interdigitated capacitor as shown in Fig. 2(c). Extra capacitance per unit length allows us to shorten the resonator length for a given resonance frequency. ${ }^{16}$ We call the resonator shown in Fig. 2(c) the first fractal iteration of the U- shaped resonator. Going further, we can create the second fractal iteration of the resonator, shown in Fig. 2(d). Obviously, it will be shorter for the same resonance frequency. Finally, we can think about the third fractal iteration of our resonator (shown in Fig. 1). All fractal iterations of the U-shaped resonator support the same type of electromagnetic mode, similar to the traditional $\lambda / 2$ resonator, while the third iteration fractal resonator is 10 times shorter than its parent resonator in Fig. 2(b).

Importantly, the fractal structure is naturally compatible with the technique of dividing the superconductor in narrow strips, widely used to increase resilience of superconducting resonators to magnetic fields. ${ }^{17}$ In our design, we use a width of $2 \mu \mathrm{m}$ for most features. This is readily achieved with standard lithography techniques, but still large enough not to be adversely affected by additional kinetic inductance and a

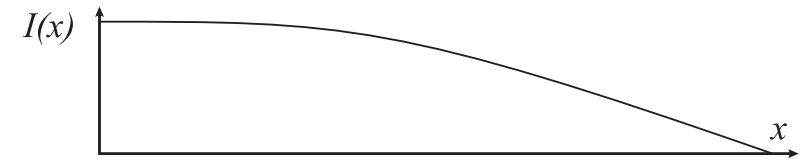

b

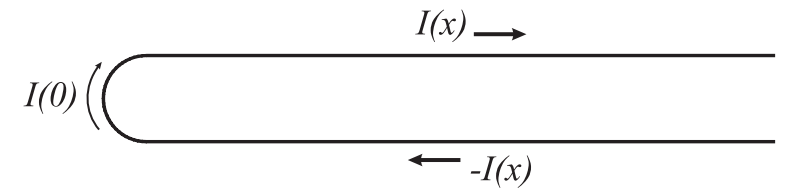

C

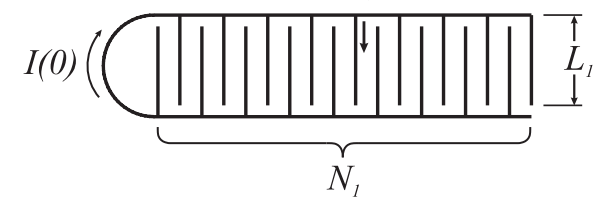

d

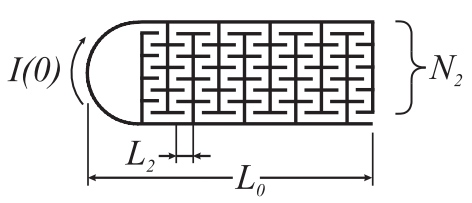

FIG. 2. (a) Current distribution along one branch in a balanced half wave resonator sketched in (b). $I(0)$ is the current where the two branches meet, i.e., the voltage node. (c) First order distributed fractal resonator of equal frequency loaded with $N_{1}$ secondary branches each carrying a current $\sim I(0) / N_{1}$. (d) 2 nd order fractal resonator where the secondary branches are split into $N_{2}$ sub-branches. For the third order fractal, used in our design, the main branch of length $L_{0}$ will be 10 times shorter for the same resonance frequency. For illustrative purposes, the relative length of the resonators is not to scale.

nonlinearities due to length scales comparable to penetration depth and coherence length in niobium, the material we use for its high critical fields. The distributed capacitance and inductance in this quasi-one-dimensional resonator are still geometrically inseparable, and the resonators support an electromagnetic resonance associated with a "slow" standing electromagnetic wave. This is contrary to similar-looking resonators studied earlier. ${ }^{18}$ There, the inductive coupling and different cavity modes were investigated for their possible use in circuit quantum electrodynamics to improve the coupling to qubits and reduce decoherence. In our case, the same argumentation can be applied albeit with the important distinction that our resonators are still distributed.

Our resonators retain an important and useful feature of traditional CPW resonators: it allows concentration of current at the antinode at $x=0$ (see Fig. 2). This feature ensures the possibility of a good coupling of spin systems to the resonator, and we also use it to inductively couple to the transmission line (see Fig. 1(b)). Despite their complex geometry, these resonators have a single mode in the range of 4-8 GHz. Due to the inductive coupling, only antisymmetric modes are excited, with the second mode being $3 \lambda / 2$. The coupling was designed to be relatively weak with $Q_{c}$ ranging from $0.6 \times 10^{5}$ up to $3 \times 10^{5}$.

\section{EXPERIMENT}

The resonators in this study were fabricated on R-plane sapphire and high resistivity silicon. After sputtering a 
$200 \mathrm{~nm}$ thick niobium film, the resonators were patterned using electron beam lithography and subsequent etching in a $\mathrm{CF}_{4}: \mathrm{O}_{2}(20: 1)$ plasma. Measurements were performed in a helium flow cryostat and a dilution refrigerator using a vector network analyzer to measure the transmitted microwave signal, $S_{21}$. Low temperature, low power measurements were done by feeding the excitation signal through heavily damped and thermalized coaxial lines. We used a low noise amplifier $\left(T_{N}=5 \mathrm{~K}\right.$ and gain $\left.+30 \mathrm{~dB}\right)$ isolated by two low temperature circulators.

Analysis was done by fitting the measured data to a skewed Lorentzian

$$
S_{21}=\frac{S_{\min }+2 i Q \delta f}{1+2 i Q \delta f}+a,
$$

where $Q=Q_{i} Q_{c} /\left(Q_{i}+Q_{c}\right)$ is the total quality factor, $\delta f$ is the normalized frequency around the center frequency, $S_{\min }=Q_{c} /\left(Q_{i}+Q_{c}\right)$ is the transmission at resonance, and $a$ is a complex asymmetry parameter accounting for some of the microwave signal by-passing the resonator. From this, the internal $\left(Q_{i}\right)$ and external $\left(Q_{c}\right)$ quality factors were extracted. Samples were zero-field cooled and all measurements in magnetic field were performed over one single sweep to avoid permanent flux trapping.

We have complemented the low temperature measurements with additional measurements done at higher power and temperature $(1.7 \mathrm{~K})$. As demonstrated and discussed in Sec. IV, both these parameters have, within this range in power and temperature, very small effect on the performance of the resonators, and we can safely extend these results to low powers and temperatures.

\section{RESULTS AND DISCUSSION}

\section{A. Magnetic field dependence of the Q-factor}

In Fig. 3, we show a typical behavior of the microwave transmission around the resonance feature as we increase the magnetic field. There is good agreement between measured data and the fit to Eq. (1) even at high magnetic fields and relatively low power.

Following the terminology of several other groups, ${ }^{9,10,13}$ we define a quality factor $Q_{B}$, associated with losses induced by magnetic field

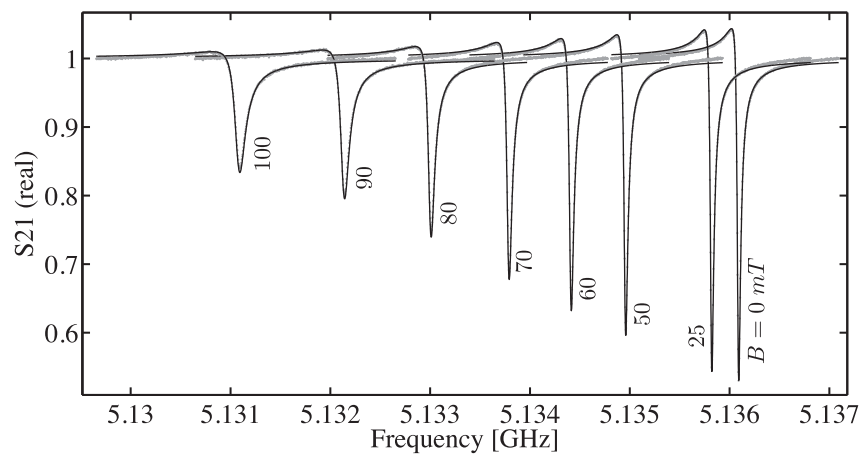

FIG. 3. Selected transmission amplitudes for one resonator measured at $-108 \mathrm{dBm}$ and a perpendicular field going from 0 to $100 \mathrm{mT}$. Black lines are fit to theory and grey dots are measured raw data.

$$
\frac{1}{Q_{B}}=\frac{1}{Q_{i}(B)}-\frac{1}{Q_{i}(B=0)}
$$

We define the magnetic field losses from the internal quality factor since it gives an intuitive understanding of the losses inside the resonator and we do not measure any noticeable variations in the coupling as we change the magnetic field.

Fig. 4(a) shows the internal quality factor $Q_{i}$ versus magnetic field applied parallel to the substrate. Fig. 4(b) presents extracted plots for $Q_{B}^{-1}$ for the two resonators in Fig. 4(a) taken at $20 \mathrm{mK}$ and two additional measurements taken at $1.7 \mathrm{~K}$. Remarkably, all data collapse onto a single curve. The measurements performed at $1.7 \mathrm{~K}$ (squares Fig. 4(b)) follow almost the same curve as the measurements performed at $20 \mathrm{mK}$, despite the large differences in temperature and measurement power. Figs. 4(c) and 4(d) present the field induced dissipation for resonators subjected to perpendicular magnetic field. At $20 \mathrm{mT}$, we maintain internal quality factors above 25000 . In the case of parallel field, the resonators can be subjected to $160 \mathrm{mT}$ for the same quality factors, sufficient for free spin ESR at $5 \mathrm{GHz}$.

From Figs. 4(b) and 4(d), it is also evident that $Q_{B}$ is power independent ${ }^{10,19}$ even down to the single photon regime. This power independence is expected in the high power regime for a narrow zero field cooled strip, ${ }^{19}$ and we show that this also holds for extremely low powers. In fact, the average number of photons in the cavities is around $\left\langle n_{\text {phot }}\right\rangle$ $\sim 5-10$ when the resonators are excited with $-143 \mathrm{dBm}$ in zero field. As we increase the magnetic field, we change $Q_{i}$ of the resonators and the occupancy is reduced to $\left\langle n_{\text {phot }}\right\rangle \sim 0.2$.

\section{B. Flux focusing, current distribution, and current branching}

A few factors contribute to the superior quality of fractal resonators in high magnetic fields. First of all, in a standard CPW design, the magnetic field expelled from the ground plane is concentrated in narrow gaps between the ground plane and the central strip - the effect known as flux focusing. For a typical coplanar design, the flux focusing factor could be as high as $500 .{ }^{12}$ For a fractal design, the flux focusing is dramatically reduced, because the non-metalized area surrounding the fractal resonator is $150 \mu \mathrm{m}$ wide, which is $\sim 30$ times wider than a gap in a typical coplanar resonator. The flux focusing can be reduced even further by replacing a solid ground plane with fractal-like structure which allows all excess flux to escape (a detailed discussion follows in Sec. IV C).

The flux focusing factor for our resonators can be estimated as follows: by scaling the data in Fig. 4(d) by a factor $\gamma \sim 8$ in magnetic field, we recover qualitatively the same curve as in Fig. 4(b). This number is to be compared against the expected scaling for the most simple geometry: a single strip of superconducting thin film. For a strip, the vortex entry field for in-plane orientation can be estimated $\mathrm{as}^{20}$ $B_{S}^{\|} \approx \phi_{0} / 2 \sqrt{2} \pi \lambda_{L} \xi$; substituting typical values ${ }^{21}$ for the London penetration depth $\lambda_{L} \approx 90 \mathrm{~nm}$ and coherence length $\xi \approx 50 \mathrm{~nm}$, we arrive at $B_{S}^{\|} \approx 50 \mathrm{mT}$. This is close to the observed onset ( $40 \mathrm{mT})$ of vortex dissipation in Fig. 4(a), where no flux focusing is expected. On the other hand, for 
(a)

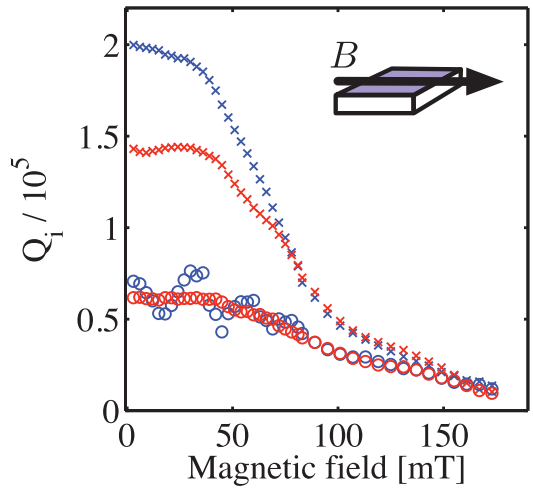

(c)

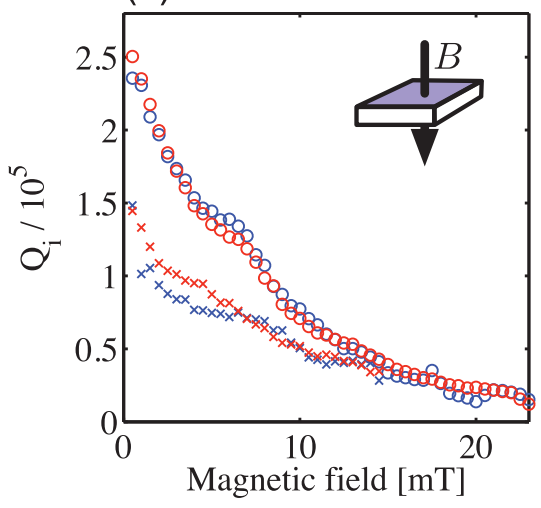

(b)

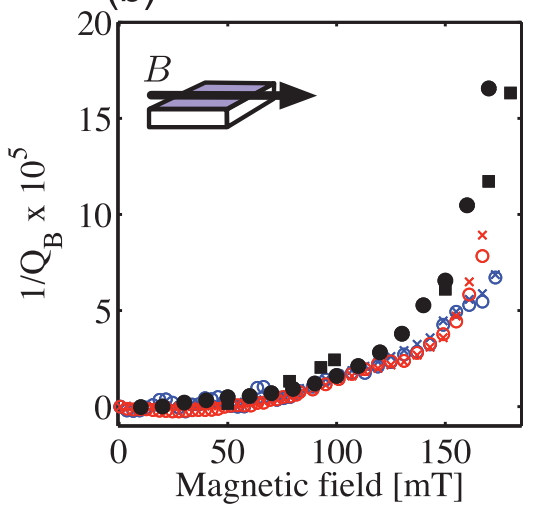

(d)

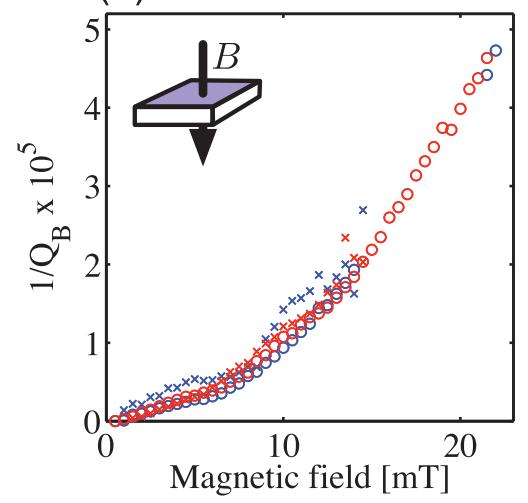

FIG. 4. (a)-(d) Resonator response to external magnetic field. Different colors indicate a different resonator. The data in this figure come from a fractal resonator with a ground configuration of type "B," discussed later in Sec. IV C. (a) Internal quality factor for in-plane magnetic field. (b) Extracted field induced energy loss $1 / Q_{B}$ for the same measurement. In both (a) and (b), circles indicate measurement performed at low power $(-143 \mathrm{dBm})$ and crosses at high power $(-108 \mathrm{dBm})$. $\square$ and (O) indicate measurements performed at $\mathrm{T}=1.8 \mathrm{~K}$ and $-70 \mathrm{dBm}$ for two resonators identical to the ones measured at $20 \mathrm{mK}$, but from a different wafer. (c) Internal quality factor for normal-to-plane orientation of the magnetic field. (d) $Q_{B}^{-1}$ for normal-to-plane orientation of the magnetic field. In both (c) and (d), applied powers are $-105 \mathrm{dBm}(\bigcirc)$ and $-70 \mathrm{dBm}(\times)$. normal to plane orientation, the expected value for the entry field is given by Ref. $17 B_{S}^{\perp} \approx \phi_{0} / 2 \pi \lambda_{L} \xi \times \sqrt{2 t / \pi L}$ $=17 \mathrm{mT}$ for a film thickness $t=200 \mathrm{~nm}$ and strip width $L=2 \mu \mathrm{m}$. If the normal to plane field is enhanced $k$ times due to flux focusing, the expected scaling factor is $\gamma \approx k B_{S}^{\|} / B_{S}^{\perp}$, and we therefore conclude that for our design $k \approx 3$, more than 100 times smaller than for a conventional coplanar design. ${ }^{12}$ Having such a small flux focusing factor removes the need for a very precise alignment of a parallel magnetic field.

Another common technique to minimize the vortexinduced dissipation is to arrange antidots or narrow open strips for trapping expelled vortices. ${ }^{11}$ The fractal structure is, in essence, an implementation of this design idea taken to extreme. All superconducting strips in fractal can easily be made $2 \mu \mathrm{m}$ wide. Moreover, while an antidot is a vortex trap encircled by a superconductor, in a fractal design all nonmetallized areas are directly connected to a much larger open space.

Finally, one can show that dissipation in the fractal resonator only happens in the main branches, the losses in higher order branches being negligible. Indeed, in the fractal design, only the main branches carry the total current. The current is then divided among the higher order branches, as illustrated in Fig. 2. If we assume that the current density is homogeneous across the section of superconducting strips (which is actually a very rough assumption that we will refine later), then the total dissipation is

$$
P_{\text {fractal }} \approx p_{0}\left[L_{0}+\frac{L_{1}}{N_{1}}+\frac{L_{2}}{N_{1} N_{2}}+\frac{L_{3}}{N_{1} N_{2} N_{3}}+\ldots\right],
$$

where $p_{0}$ is the dissipation per unit length of a fractal line and $N_{k}$ is the number of sub-branches of the k-th order. In this particular design for a center frequency of $5 \mathrm{GHz},{ }^{25}$ we have $N_{k}=[12,6,8]$, and the length of the respective segments $L_{0} \approx 1 \mathrm{~mm} \gg L_{1}, L_{2}, L_{3}$. It is evident that the major contributions to dissipation come from the first term in Eq. (3). Contributions from higher order terms are in our case $\sim 5 \%$.

This simple estimation is supported by our numeric simulation presented in Fig. 5. It shows that the current amplitude in the 4 th-order branches is at least $20 \mathrm{~dB}$ below the one in the primary branches.

To estimate the vortex related dissipation in the main branches, we shall consider the current density $j(x)$ and flux density $B(x)$ distribution across the section of the primary branch. The dissipation is proportional to ${ }^{13} \int_{L / 2}^{L / 2} B(x) j^{2}(x) d x$, where $L=2 \mu \mathrm{m}$ is the width of the strip.

To this end, we note first that for an ideal $(\sigma \rightarrow \infty)$ conductor, the simulated current density distribution in the main branch is essentially the same as for a single strip (see Fig. 5). Although we do not have a rigorous proof for this statement, physically it is clear that for any n-order branch carrying some current $I_{n}$ there exists a neighboring branch carrying an opposite current $-I_{n}$, next to which another neighboring branch carrying again $I_{n}$ and so on. On a large scale, this means that the dipole momentum of the total magnetic field from high order branches is compensated. As a result, the whole fractal structure filling the space between the primary branches has essentially no effect on the current distribution in the primary branches. This result, together with Eq. (3), reduces the problem of dissipation in a fractal 

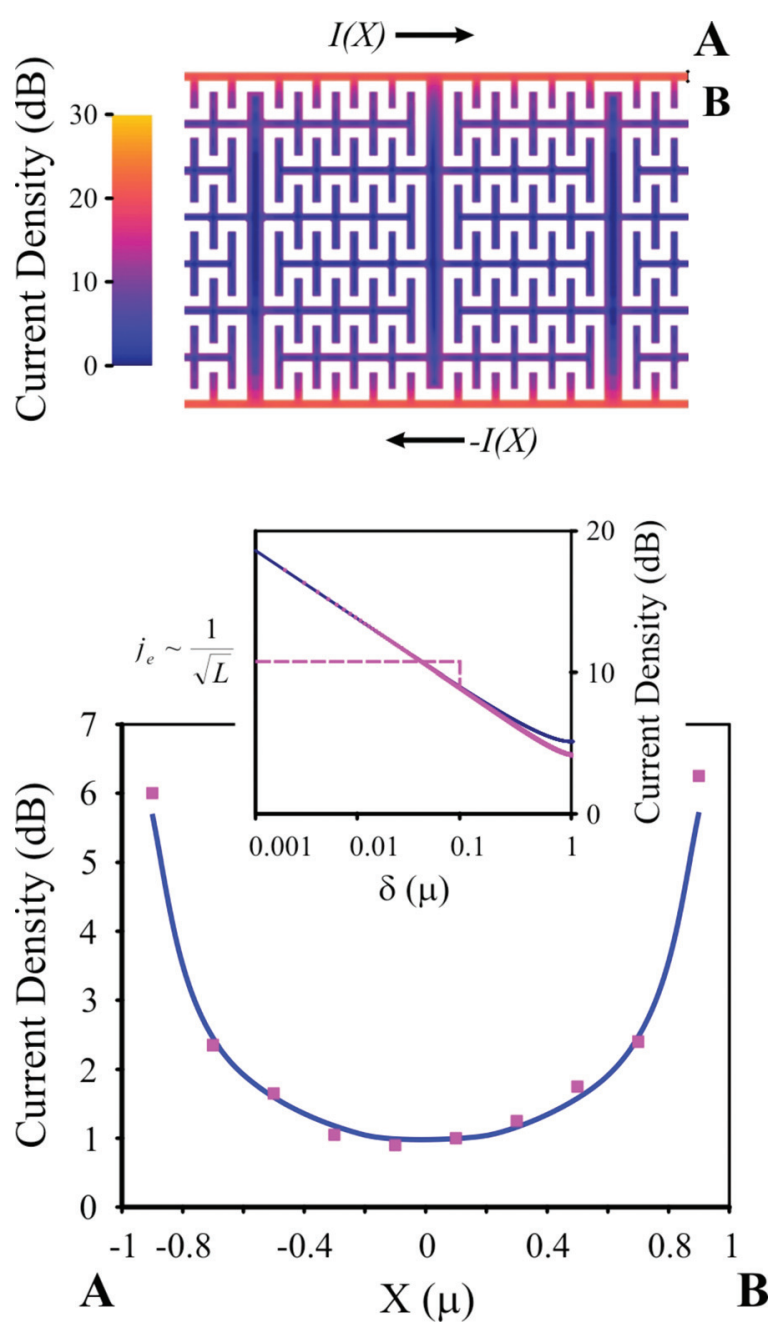

FIG. 5. Numerically calculated current distribution in a segment of the fractal resonator. Simulations were done using AWR Microwave Office and the EMsight simulator. Bottom: Current density along the cross-section of the fractal main branch (points A to B in the top panel). Points are extracted from simulation and solid line is calculated using Eq. (4). Inset: Current density at a distance $\delta$ from the edge of a single strip (blue) and an equivalent coplanar strip (purple) for an ideal conductor. Dashed line indicates the simplest model for a superconductor with a constant density up to the penetration depth $\lambda_{L}$.

resonator to the much easier case of dissipation in a single superconducting strip.

The current density in a single strip of an ideal conductor is obtained through conformal mapping

$$
j(x)=\frac{2 I}{\pi L} \frac{1}{\sqrt{1-(2 x / L)^{2}}},
$$

where $I$ is the total current. As our final goal is to compare dissipation in our resonators to a standard CPW design, we shall compare Eq. (4) to the current density in the central strip of a coplanar resonator ${ }^{19}$

$$
j(x)=\frac{I}{L K(L / a)} \frac{1}{\sqrt{1-(2 x / L)^{2}} \sqrt{1-(2 x / a)^{2}}},
$$

where $K$ is the complete elliptic integral and $a=L+2 W$ and $W$ is the gap between central strip and the ground plane.
The first square-root in Eq. (5) is divergent at the strip edge, while the second is not. This means that asymptotically (close to the edge) the current distribution in a single strip is the same as in slightly wider coplanar resonator. For a strip $2 \mu \mathrm{m}$ wide, the edge current in a remote strip is the same as in a coplanar resonator of width $2.3 \mu \mathrm{m}$ (assuming a line impedance of $50 \Omega$ ), see inset in Fig. 5 .

However, the edge current divergency in Eq. (5) stems from the fact that an ideal conductor completely expels the microwave field, while for a superconductor the magnetic field penetrates into the material on the scale of the London penetration depth $\lambda_{L}$. This smears the magnetic field and eliminates the current divergency. The simplest model for a superconducting strip thus simply presumes the edge current density $j_{e}$ to be constant up to the depth $\lambda_{L}$ (see Fig. 5): ${ }^{22}$

$$
j_{e}(x)=\frac{I}{L K(L / a)} \frac{1}{\sqrt{\frac{\lambda_{L}}{L}\left(1-(L / a)^{2}\right)}} .
$$

Given the current distribution (5)-(6) and a known flux density in a superconducting strip (considered by Norris ${ }^{23}$ and later refined by Brandt and Indenbom, ${ }^{24}$ and together referred to as the NBI model), we can, in principle, write explicit formulas for the dissipation. But to make a comparison to a standard CPW design, it is sufficient to establish how the dissipation scales when we move from a CPW with a central strip $2.3 \mu \mathrm{m}$ wide (which, as discussed, is equivalent to our fractal design) to a common dimension of $10 \mu \mathrm{m}$. We note first that the NBI model predicts that the flux density is some universal function of the dimensionless coordinate $x / L$ : $B=B(x / L)$. For a given wave impedance of a CPW line, the dissipation in the edge $\left(\lambda_{L}\right)$ region does not depend on $\mathrm{L}$

$$
P_{e}=\int_{0}^{\lambda_{L}} B(x / L) j_{e}^{2} d x \sim I^{2} \int_{0}^{\lambda_{L}} B(x / L) d(x / L) .
$$

And dissipation in the bulk scales as $L^{-1}$

$$
P_{b} \sim \frac{I^{2}}{L} \int_{-L / 2+\lambda_{L}}^{L / 2-\lambda_{L}} B(x / L) \frac{1}{1-(2 x / L)^{2}} d(x / L) .
$$

This implies that if the edge dissipation dominates (i.e., for $B \rightarrow 0$ ), the dissipation per unit length for a fractal structure is the same as for a CPW resonator (assuming a hypothetic case of no flux focusing for the latter). As our fractal resonator is 12 times shorter than a CPW with the same resonance frequency, we see that for a fractal design the low field dissipation is reduced by an order of magnitude.

In a strong magnetic field, when vortices penetrate the superconductor deeper than by $\lambda_{L}$, the per unit length dissipation in a fractal line is $\sim 4$ times higher than for a traditional $10 \mu \mathrm{m}$ wide $\mathrm{CPW}$, but the total dissipation is still $12 / 4=3$ times lower.

Using similar argumentation, we can qualitatively explain the behavior of the loss rate observed in Fig. 4(d). At low fields, the flux density (and also the dissipation) scales linearly with field just at the edge; however, the flux penetration depth is also increasing with magnetic field so that the total 
dissipation scales with a higher power of $B$. The exact scaling power depends on the microscopic edge profile of the strip. ${ }^{24}$ Once the flux has penetrated deep inside the strip we recover a linear dependence of the dissipation with magnetic field, in accordance with the previous studies of type II resonators. ${ }^{11,13}$ The only difference being that for a narrow strip the initial region with a strong magnetic field dependence becomes more pronounced.

\section{Ground plane optimization}

In a conventional coplanar design, nearly half of the total power dissipation takes place in the ground planes. In this sense, the geometry of our resonators also serves to reduce the magnitude of the induced current, since the "prong-to-prong" capacitance $C_{p-p}$ is much larger than the "prong-to-ground" capacitance $C_{p-g}$. In fact, $C_{p-p} / C_{p-g} \approx 10^{3}$ for our particular design. This means that the currents in the ground plane become very small and the energy dissipated here becomes negligible. The ground plane will, however, affect the flux focusing and the local magnetic field experienced by the resonator. Additionally, the ground planes are needed to localize the electric field from the resonator and cannot be completely removed without introducing significant zero-field losses. To find the optimal trade-off between these two extremes and to maximize the performance of the resonators in magnetic field, we have investigated how various types of ground plane designs influence the magnetic field associated losses of the resonator.

Conceptually, these variations are shown in Fig. 6. We will hereafter refer to these different designs by their

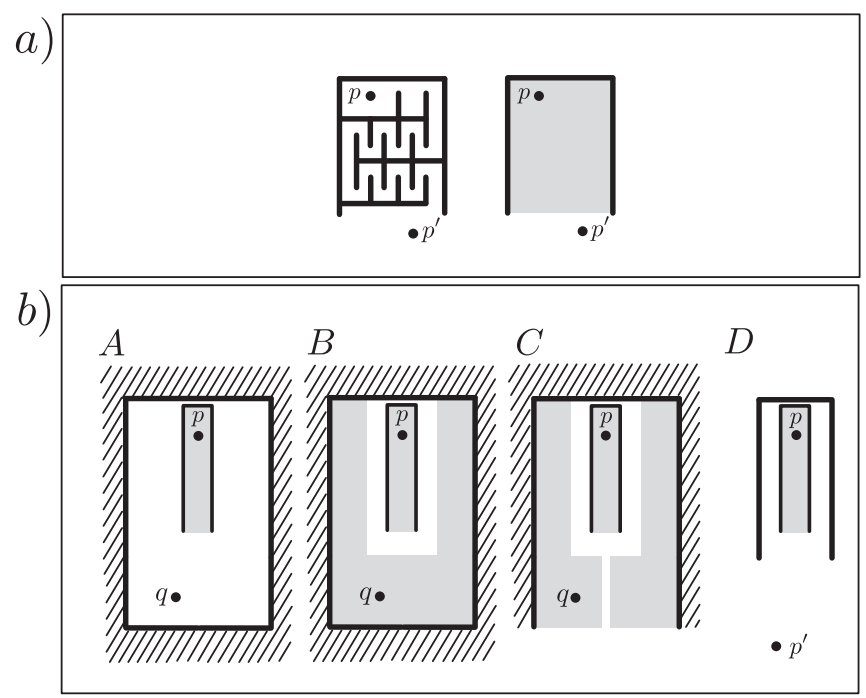

FIG. 6. (a) Left: The topology of the fractal resonator. For any point p inside the fractal there exists a path to the point $\mathrm{p}^{\prime}$ outside the structure such that no superconducting segment needs to be crossed. Right: Graphical representation of the same topology as in the left figure, used in (b). The grey area represents the fractal structure. (b) Designs of various resonator/ground configurations discussed in the text. Hatched areas indicate larger unpatterned ground planes and solid lines indicate a continuous path of superconductor. There exists a path $p_{C / D}-p^{\prime}$ for topologies $\mathrm{C}$ and $\mathrm{D}$. In topology $\mathrm{C}$, there is also a path $q_{C}-p$ ' for any point $q_{C}$ in the ground plane, whereas in design $\mathrm{D}$ the ground plane is reduced to a small segment just around the resonator. The designs are sorted alphabetically in order of their performance (Q) in parallel magnetic fields above $100 \mathrm{mT}$, such that Q increases from A to D. corresponding labeling in Fig. 6(b). All designs have the same fractal resonator structure, and are made on the same wafer under the same conditions. Design A has most of the ground plane removed, but there is still a continuous path of superconductor around the resonator. That is, it is not possible to connect points $p_{A}$ and $p^{\prime}$ in Fig. 6(b) without crossing a segment of superconductor. Design B has the empty area in A filled with a fractal-like structure, such that the electromagnetic screening of the resonator is improved. In design $\mathrm{C}$ (the design shown in Fig. 1), the ground loop is broken so that excess flux can easily escape. Here, there is a path connecting any non-metalized point $q_{C}$ inside the fractal ground and the point $\mathrm{p}^{\prime}$. In design $\mathrm{D}$, the ground plane is completely removed, except for a thin strip around the resonator used to localize electric fields. The feeding line has also been removed, the resonator is directly coupled by placing the silicon chip on a copper PCB with a transmission-line.

We measured the field induced loss rates for these ground plane designs. The result is shown in Fig. 7 and we have extracted the magnetic fields for which $Q_{B}=10^{5}$ and $2 \times 10^{4}$ in Table I for an easier comparison.

As expected, removing the ground planes serves to increase the resilience of the resonators to magnetic field. However, removing all ground will spread out the electric fields outside the resonator, and obviously $Q_{i}$ will drop due to radiation losses and interaction with a large portion of the substrate. We find that a fractal-like geometry of the ground plane (C) provides a good trade-off. It will make sure there is no trapped flux around the resonator and at the same time it provides good screening such that the electric fields and radiation losses are kept relatively small. Additionally, any currents induced in these ground planes will lead to less dissipation based on the same arguments as for the resonator itself.

In the best performing design $(\mathrm{C})$, the ground plane around the resonator is similar to the structure of the

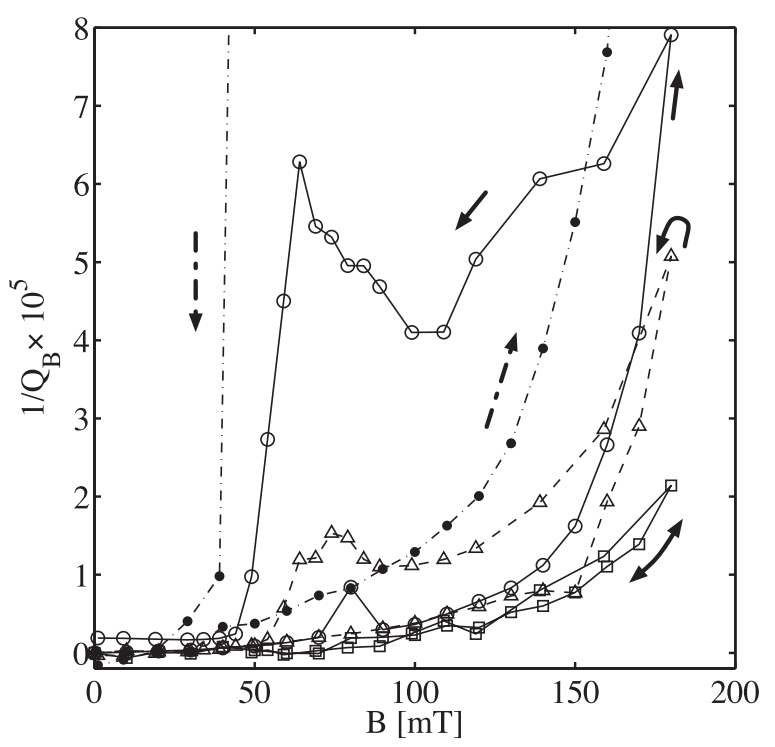

FIG. 7. Magnetic field associated loss rates in parallel field for the four different topologies shown in Fig. 6. $(\bigcirc)=$ Topology $A,(\bigcirc)=B,(\Delta)=C$, $(\square)=\mathrm{D}$. We also show the hysteretic behavior observed when returning the field to zero. 
TABLE I. Summary of resonator performance in magnetic field for the different ground plane designs in Fig. 6. Numbers are the mean values taken over several devices. In most cases, $2-4$ resonators have been measured from each sample, and for several cases we have also measured more than one sample. All samples come from the same wafer. In all cases, the deviation from these values between devices is essentially the same. For internal Q, the deviation is about $\pm 10 \%$, and for magnetic fields around $\pm 5 \%$.

\begin{tabular}{lccc}
\hline \hline Topology & $Q_{i}(B=0)$ & $\begin{array}{c}\text { B-field where } \\
Q_{B}=1 \times 10^{5}(\mathrm{mT})\end{array}$ & $\begin{array}{c}\text { B-field where } \\
Q_{B}=2 \times 10^{4}(\mathrm{mT})\end{array}$ \\
\hline A & 34200 & 93 & 145 \\
B & 103500 & 137 & 173 \\
C & 83300 & 148 & 177 \\
D & 37100 & 157 & 201 \\
\hline \hline
\end{tabular}

resonator itself. We deem this design to be the best one for our target application despite that in Fig. 7 design D shows even lower values for $Q_{B}^{-1}$. The reason is that design $\mathrm{D}$ has a much lower internal zero field $\mathrm{Q}$, and for the relevant magnetic fields measured, this internal loss rate is always dominating. However, in the end, the optimal design depends on the target field for the specific application.

For all designs in Fig. 7, we also show the hysteretic behavior as we return the magnetic field to zero. We observe that there is almost no hysteresis at all for the fractal design up to some specific magnetic field $B_{\alpha}$. This is shown in Fig. 7 for design D. We see the same behavior for the other designs as well, but at slightly lower threshold fields $B_{\alpha}$. When $B>B_{\alpha}$, the resonator enters a dissipative state. It will remain in this state even for fields $B<B_{\alpha}$ until some other threshold occurs at $B_{\beta}<B_{\alpha}$. At this point, the original Q is almost immediately recovered. In accordance with Ref. 13, there is hysteresis at high fields, but as we return to zero field we recover the same loss rates as in the initial state (Fig. 7). In fact, in most of our samples, the resonators returned to exactly the same state. Typical variations are on the order of a few percent, which, at least to some extent, can be attributed to noise in the measurement, fitting errors, and temperature drift. The difference compared to Ref. 13 most likely originates in the fractal geometry, and in perpendicular field our observed hysteretic behavior cannot be fully explained by the model given in Ref. 13. This suggests more complicated vortex dynamics in the fractal design. However, this will be a topic for further research.

\section{Power dependence}

One potential drawback of the fractal design is an increased perimeter. This could potentially lead to coupling to a larger number of defects and two-level fluctuators (TLFs) in the materials involved. For this reason, we measured the power dependence of two resonators, plotted in Fig. 8, to verify that the increased surface area of the fractal design does not significantly affect the behavior of the resonator. We observe a typical reduction in the quality factor with excitation powers. The lowest measured power in our case corresponds to $\sim 0.1$ photons on average in the cavity. As we approach single photon numbers, we observe a saturation in the quality factor. This can be explained by assuming

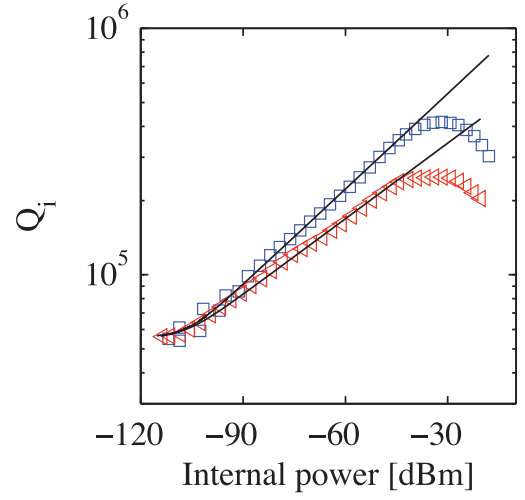

FIG. 8. Internal quality factor for two resonators (same as in Fig. 4) as a function of equivalent internal power in the resonator measured at $20 \mathrm{mK}$ at zero magnetic field. Black lines are fits to TLF theory according to Eq. (9).

that the cavity is coupled to an ensemble of weakly coupled TLFs. As we increase the excitation power, we saturate these TLFs and the Q-factor increases. In general, the power dependence in the linear regime can be described as ${ }^{6,7}$

$$
Q\left(P_{\text {res }}\right)=\left(1+\frac{P_{\text {res }}}{P_{0}}\right)^{\alpha}, \quad P_{\text {res }}=\frac{2}{\pi} \frac{Z_{0}}{Z_{r}} \frac{Q^{2}}{Q_{c}} P_{\text {exc }},
$$

where $P_{\text {res }}$ is the equivalent power of the voltage standing wave inside the resonator, $P_{0}$ is a characteristic power below which the TLFs remain in the ground state; $\alpha$ is material and geometry dependent and describes the interaction of the photon field with the TLFs. In our case, we find $\alpha=0.10$ and 0.13 for two resonators on the same substrate. These are relatively large numbers, an indication of large variations of electric field distribution across the resonant structure and interaction with many differently coupled TLFs. ${ }^{7,26}$ The relatively low saturation quality factor also indicates that a large number of TLFs are involved, as expected from the fractal geometry. However, the magnetic field induced loss rates will still be dominating in this structure at the magnetic fields required for free spin interaction. But for further improvements and operation in the single photon regime, this is an issue that may need to be addressed.

\section{E. ESR spectrometry}

Fig. 9 shows the measured dissipation for a resonator on which we have placed a small flake of a 2,2-diphenyl1-picrylhydrazyl (DPPH) crystal. DPPH is an organic molecule that is commonly used in ESR as a reference for its simple spectrum. We estimate this flake to contain $10^{11}$ $-10^{12}$ molecules, each having a free radical. The measurement was performed at high temperature $(1.7 \mathrm{~K})$. When the Zeeman splitting of the free spins is equal to the frequency of our resonator (here, $3.73 \mathrm{GHz}$ ), we observe a large increase in the dissipation.

This measurement proves that the resonator described in this paper is suitable for ESR spectroscopy; it maintains all important properties of a distributed resonator and it is still possible to achieve a strong coupling with the spin system. While improving the magnetic field properties of our superconducting resonator, we have also addressed several other 


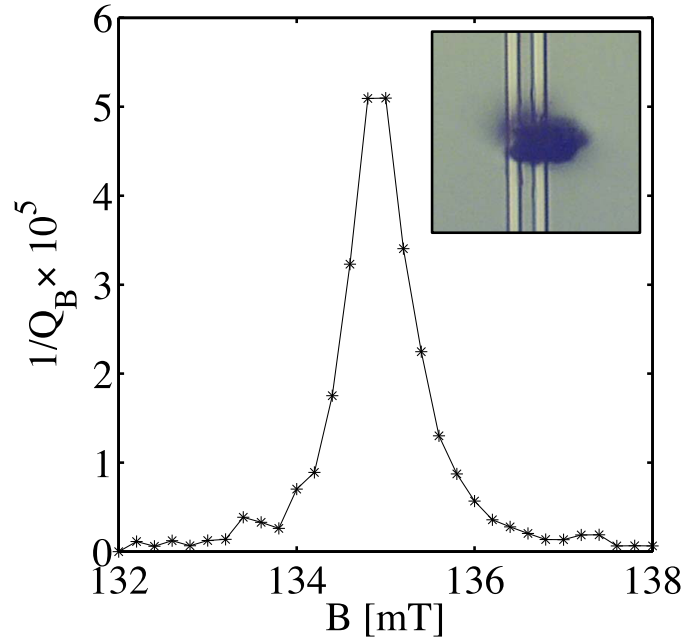

FIG. 9. Measured ESR signal near the Zeeman field $B=h \nu / 2 \mu_{B}$ from a small flake of a DPPH crystal at $T=1.7 \mathrm{~K} . Q_{B}^{-1}$ is normalized to $132 \mathrm{mT}$ to illustrate the additional dissipation channel introduced by the spins. Inset: Optical image of the DPPH flake coupled to two strips of the resonator near the current antinode, the same area is indicated in Fig. 1. Strip widths are $2 \mu \mathrm{m}$.

issues important for this type of measurement. The narrow width of the superconducting strips naturally increases the coupling, although at the same time this also limits to a volume set by the extension of the rf-magnetic field around the strips. Furthermore, since all ground planes close to the resonator are removed, the static magnetic field will be much more homogeneous, resulting in a reduced line-width of the spin ensemble.

\section{CONCLUSIONS}

In summary, we have demonstrated a universal approach to reducing magnetic field induced loss in superconducting thin film resonators. Using a fractal network in conjunction with several previously known methods to increase resilience to magnetic fields, we observe a large increase in the quality factors. We measure internal quality factors above 25000 in magnetic fields corresponding to the Zeeman splitting equivalent to the operating frequency of these resonators. We attribute the increased quality factors mainly to the current branching in the fractal geometry of our resonators.

We expect it to be possible to increase the tolerance to magnetic fields even further by increasing the order of the fractal, reducing the length of the dissipative branch even more. Reducing the thickness of the $\mathrm{Nb}$ has also been shown to yield improved magnetic field properties. ${ }^{27}$

As also demonstrated, we maintain all important properties of a distributed resonator when it comes to the coupling of a spin ensemble to the resonator, and we are able to measure an ESR signal from a microscopic volume of free spins. This significant improvement opens up for the detection and interaction with a very small number of free spins. We believe that our results are important for a broad range of applications involving superconducting resonators, including applications in high magnetic fields.

The authors would like to thank F. Lombardi and A. Ya. Tzalenchuk for useful discussions. We acknowledge EU FP7 programme under the grant agreement ELFOS', the Marie Curie Initial Training Action (ITN) Q-NET 264034, the Swedish Research Council VR, and the Chalmers Nanoscience and Nanotechnology Area of Advance for financial support.

${ }^{1}$ J. Clarke and F. K. Wilhelm, Nature 453, 1031 (2008).

${ }^{2}$ M. Göppl, A. Fragner, M. Baur, R. Bianchetti, S. Filipp, J. M. Fink, P. J. Leek, G. Puebla, L. Steffen, and A. Wallraff, J. Appl. Phys. 104, 113904 (2008).

${ }^{3}$ M. Devoret, S. Girvin, and R. Schoelkopf, Ann. Phys. 16, 767 (2007).

${ }^{4}$ Y. Kubo, F. R. Ong, P. Bertet, D. Vion, V. Jaques, D. Zheng, A. Dréau, J. F. Roch, A. Auffeves, F. Jelezeko, J. Wrachtrup, M. F. Barthe, P. Bergonzo, and D. Esteve, Phys. Rev. Lett. 105, 140502 (2010).

${ }^{5}$ D. I. Schuster, A. P. Sears, E. Ginossar, L. DiCarlo, L. Frunzio, J. J. L. Morton, H. Wu, G. A. D. Briggs, B. B. Buckley, D. D. Awschalom, and R. J. Schoelkopf, Phys. Rev. Lett. 105, 140501 (2010).

${ }^{6} \mathrm{H}$. Wang, H. Hofheinz, J. Wenner, M. Ansmann, R. C. Bialczak, M. Lenander, E. Lucero, M. Neeley, A. D. O'Connell, D. Sank, M. Weides, A. N. Cleland, and J. M. Martinis, Appl. Phys. Lett. 95, 233508 (2009).

${ }^{7}$ P. Masha, S. H. W. van der Ploeg, G. Oelsner, E. Il'ichev, H. G. Meyer, S. Wünsch, and M. Siegel, Appl. Phys. Lett. 96, 062503 (2010).

${ }^{8}$ T. Lindström, J. E. Healey, M. S. Colcough, C. M. Muirhead, and A. Y. Tzalenchuk, Phys. Rev. B 80, 132501 (2009).

${ }^{9}$ C. Song, T. W. Heitmann, M. P. DeFeo, K. Yu, R. McDemott, M. Neeley, J. M. Martinis, and B. L. T. Plourde, Phys. Rev. B 79, 174512 (2009).

${ }^{10}$ D. Bothner, T. Gaber, M. Kemmler, D. Koelle, and R. Kleiner, Appl. Phys. Lett. 98, 102504 (2011).

${ }^{11}$ D. Bothner, C. Clauss, E. Koroknay, M. Kemmler, T. Gaber, M. Jetter, M. Scheffler, P. Michler, M. Dressel, D. Koelle, and R. Kleiner, Appl. Phys. Lett. 100, 012601 (2012).

${ }^{12}$ J. E. Healey, T. Lindström, M. S. Colclough, C. M. Muirhead, and A. Ya. Tzalenchuk, Appl. Phys. Lett. 93, 043513 (2008).

${ }^{13}$ D. Bothner, T. Gaber, M. Kemmler, D. Koelle, and R. Kleiner, Phys. Rev. B 86, 014517 (2012).

${ }^{14}$ C. Song, M. P. DeFeo, K. Yu, and B. L. T. Plourde, Appl. Phys. Lett. 95, 232501 (2009).

${ }^{15}$ N. Pompeo and E. Silva, Phys. Rev. B 78, 094503 (2008).

${ }^{16}$ C. E. Gough, A. Porch, M. J. Lancaster, R. J. Powell, B. Avenhaus, J. J. Wingfield, D. Hung, and R. G. Humphreys, Physica C 282-287, 395 (1997).

${ }^{17}$ D. Yu. Vodolazov and I. L. Maksimov, Physica C 349, 125 (2000).

${ }^{18}$ M. S. Khalil, F. C. Wellstood, and K. D. Osborn, IEEE Trans. Appl. Supercond. 21, 879 (2011).

${ }^{19} \mathrm{P}$. Lahl and R. Wördenweber, Appl. Phys. Lett. 81, 505 (2002).

${ }^{20}$ C. P. Bean and J. D. Livingston, Phys. Rev. Lett. 12, 14 (1964).

${ }^{21}$ A. I. Gubin, K. S. Il'in, S. A. Vitusevich, M. Siegel, and N. Klein, Phys. Rev. B 72, 064503 (2005).

${ }^{22}$ Note: Allthough more refined models are known from the literature, for the sake of this qualitative discussion it is enough to stay with the simplest model.

${ }^{23}$ W. T. Norris, J. Phys. D 3, 489 (1970).

${ }^{24}$ H. Brandt and M. Indenbom, Phys. Rev. B 48, 12893 (1993).

${ }^{25}$ Note: The perimeter of the fractal is about $12 \mathrm{~mm}$, so that the total capacitance $C=1.1 \mathrm{pF}$ is similar to a CPWR with the same fundamental frequency. For our particular design, we have a total inductance $\sim 1 \mathrm{nH}$ and wave impedance $Z_{r} \approx 30 \Omega$.

${ }^{26}$ J. Wenner, R. Barends, R. C. Bialczak, Yu. Chen, J. Kelly, E. Lucero, M. Mariantoni, A. Megrant, P. J. J. O’Malley, D. Sank, A. Vainsencher, H. Wang, T. C. White, Y. Yin, J. Zhao, A. N. Cleland, and J. M. Martinis, Appl. Phys. Lett. 99, 113513 (2011).

${ }^{27}$ J. H. Quateman, Phys. Rev. B 34, 1948 (1986). 